OPEN ACCESS

Edited by:

Claire Booth,

University College London,

United Kingdom

Reviewed by:

Katrina Louise Randall,

Australian National University, Australia

Alessandro Aiuti,

Vita-Salute San Raffaele University,

Italy

*Correspondence: Yulia Rodina

r.j.doctor77@gmail.com

Specialty section:

This article was submitted to Primary Immunodeficiencies,

a section of the journal

Frontiers in Immunology

Received: 02 May 2021

Accepted: 23 August 2021 Published: 09 September 2021

Citation:

Rodina Y, Deripapa E, Shvets O, Mukhina A, Roppelt A, Yuhacheva D, Laberko A, Burlakov V, Abramov D,

Tereshchenko G, Novichkova $G$ and

Shcherbina A (2021) Rituximab

and Abatacept Are Effective in

Differential Treatment of Interstitial

Lymphocytic Lung Disease in Children

With Primary Immunodeficiencies.

Front. Immunol. 12:704261.

doi: 10.3389/fimmu.2021.704261

\section{Rituximab and Abatacept Are Effective in Differential Treatment of Interstitial Lymphocytic Lung Disease in Children With Primary Immunodeficiencies}

Yulia Rodina ${ }^{1 *}$, E. Deripapa ${ }^{1}$, O. Shvets ${ }^{1}$, A. Mukhina $^{1}$, A. Roppelt ${ }^{1}$, D. Yuhacheva ${ }^{1}$, A. Laberko ${ }^{1}$, V. Burlakov' ${ }^{1}$, D. Abramov ${ }^{2}$, G. Tereshchenko ${ }^{3}$, G. Novichkova ${ }^{4}$ and Anna Shcherbina ${ }^{1}$

\begin{abstract}
1 Department of Immunology, Dmitry Rogachev National Medical Research Center of Pediatric Hematology, Oncology and Immunology, Moscow, Russian, ${ }^{2}$ Department of Pathology, Dmitry Rogachev National Medical Research Center of Pediatric Hematology, Oncology and Immunology, Moscow, Russian, ${ }^{3}$ Department of Radiology, Dmitry Rogachev National Medical Research Center of Pediatric Hematology, Oncology and Immunology, Moscow, Russian, ${ }^{4}$ Department of Hematology, Dmitry Rogachev National Medical Center of Pediatric Hematology, Oncology and Immunology, Moscow, Russian
\end{abstract}

Background: Interstitial lymphocytic lung disease (ILLD), a recently recognized complication of primary immunodeficiencies (PID), is caused by immune dysregulation, abnormal bronchus-associated lymphoid tissue (BALT) hyperplasia, with subsequent progressive loss of pulmonary function. Various modes of standard immunosuppressive therapy for ILLD have been shown as only partially effective.

Objectives: To retrospectively evaluate the safety and efficacy of abatacept or rituximab in treatment of ILLD in children with PID.

Methods: 29 children (median age 11 years) with various forms of PID received one of the two therapy regimens predominantly based on the lesions' immunohistopathology: children with prevalent B-cell lung infiltration received rituximab $(n=16)$, and those with predominantly T-cell infiltration received abatacept $(n=17)$. Clinical and radiological symptoms were assessed using a severity scale developed for the study.

Results: The targeted therapy with abatacept $(A)$ or rituximab $(R)$ enabled long-term control of clinical (A $3.4 \pm 1.3$ vs. $0.6 \pm 0.1 ; R 2.8 \pm 1$ vs. $0.7 \pm 0.05, p<0.01$ ) and radiological (A $18.4 \pm 3.1$ vs. $6.0 \pm 2.0 ; R 30 \pm 7.1$ vs. $10 \pm 1.7, p<0.01$ ) symptoms of ILLD in both groups and significantly improved patients' quality of life, as measured by the total scale (TS) score of $57 \pm 2.1$ in treatment recipients vs. $31.2 \pm 1.9$ before therapy $(p<0.01)$.

Conclusions: ILLD histopathology should be considered when selecting treatment. Abatacept and rituximab are effective and safe in differential treatment of ILLD in children.

Keywords: ILLD, primary immunodeficiency, children, treatment, rituximab, abatacept 


\section{HIGHLIGHTS}

1. ILLD histopathology must be considered when choosing a treatment.

2. Targeted immunosuppressive therapy is effective in achieving control of ILLD in patients with inborn errors of immunity

\section{INTRODUCTION}

Primary immunodeficiencies (PID) comprise a heterogeneous group of more than 400 inherited conditions with associated immune dysfunctions (1). Although severe recurrent/chronic infections are the main cause of mortality and morbidity in PID, in recent years, research has focused on immune dysregulation manifesting with oncological and autoimmune or autoinflammatory conditions involving various organs and systems $(2,3)$.

Interstitial lymphocytic lung disease (ILLD) is a recently characterized nonmalignant PID complication (4). In a cohort of adult patients who had common variable immunodeficiency (CVID), the incidence of ILLD was reported as 22\% (5) and as high as 38-52\% in monogenic immune dysregulation syndromes such as CTLA4 (6) and LRBA (7) deficiencies. Prophylactic antibacterial therapy and immunoglobulin (IG) replacement, although necessary to control infections, seem to have little effect on the risks of ILLD development $(8,9)$. Immune dysregulation in ILLD causes reactive bronchusassociated lymphoid tissue (BALT) hyperplasia (10), which manifests in several pathomorphological forms: follicular bronchiolitis (FB), nodular lymphoid hyperplasia (NLH), and lymphocytic interstitial pneumonia (LIP) (11). While asymptomatic at first, ILLD leads to progressive pulmonary fibrosis and eventual respiratory failure (12). Treatment of ILLD using various immunosuppressive drugs, including corticosteroids, azathioprine, cyclosporine A, cyclophosphamide, hydroxychloroquine, methotrexate, mycophenolate mofetil, and infliximab, leads to variable results ranging from partial/transient effects to no effects at all and has often been associated with adverse effects and increased rates of infection (13-17). Therefore, targeted ILLD therapy is needed. In small cohorts of adult PID patients, rituximab in combination with azathioprine has proven effective (18, 19). Recent studies have shown abatacept to be effective in controlling symptoms of LRBA and CTLA deficiency, including ILLD $(20,21)$. Yet, there have been few reports and no consensus on ILLD treatment, especially in children.

Herein, we present a retrospective analysis of the safety and efficacy of ILLD monotherapy with rituximab or abatacept, chosen based predominantly on the pathomorphological characteristics of lymphoid infiltration, as well as genetic defects, in a cohort of pediatric patients with PID.

\section{MATERIALS AND METHODS}

\section{Study Overview}

We reviewed the charts of patients with PID and ILLD who had been treated at our institution between April 2014 and October
2020 and extracted demographic, immunologic, pathomor phological, and radiographic data. We also queried the patients' charts for previous immunosuppressive therapy. In all cases, the diagnosis of PID had been made according to the ESID diagnostic criteria (22), and in most cases it had been confirmed genetically (Table E1, Supplementary Material).

The study's inclusion criteria were histological and/or radiological diagnosis of ILLD, treatment with rituximab or abatacept for at least 12 months, no signs of ongoing infectious pulmonary process at the beginning of treatment, and regular IG substitution and trough IgG levels $>5 \mathrm{~g} / \mathrm{l}$. The exclusion criteria were lack of adherence to the therapy and the use of additional immunosuppression during the period analyzed.

Included in the study were the charts of 29 patients ( 9 boys and 20 girls) who had the following PID diagnoses: ataxia telangiectasia (AT) with the ATM gene defect (8/29), CTLA4 deficiency (CTLA4) (4/29), combined immunodeficiency (CID) without genetic verification (8/29), del22 syndrome $(3 / 29)$, NFkB1/2 defect (NFkB1/2) (2/29), LRBA deficiency (LRBA) (2/ 29), Nijmegen syndrome (NBS) (1/29), Artemis deficiency (DCLRE1C) (1/29) (Table E1, Supplementary Material).

The median age at the beginning of treatment was $11.0(3-17)$ years. The median age at the first clinical signs of ILLD was 7 (2.5-14) years.

The efficacy of the ILLD treatment was assessed after 12 months of treatment. In patients who continued the treatment for more than 14 months, a long-term follow-up assessment was performed at the time of data censoring (October 2020) or at the time of hematopoietic stem cell transplantation (HSCT).

\section{Preceding Therapy}

According to the inclusion criteria, during the period analyzed, all patients had received regular IG replacement $(0.4-0.6 \mathrm{~g} / \mathrm{kg}$ every 4 weeks) with a mean trough IgG level of $7.67 \pm 0.66 \mathrm{~g} / \mathrm{l}$ (5.15-20 g/l).

Of the 29 patients, 17 had previously received immunosuppressive therapy for ILLD or other immune complications (Table E1, Supplementary Material) using azathioprine (3), mycophenolate mofetil (5), sirolimus (3) or adalimumab (1), methotrexate (2), and rituximab (2), either with no or only partial resolution of the ILLD symptoms. In addition, 14 patients also received corticosteroids (1-2 $\mathrm{mg} / \mathrm{kg} /$ day) for 1-3 months and experienced the resolution of some lesions. The corticosteroid therapy had been accompanied by the following side effects: Cushing syndrome (14/14), osteopenia (10/14), and systemic bone loss (2/14). After cessation of corticosteroid therapy, all 14 patients had experienced ILLD relapse/progression.

\section{ILLD Severity Evaluation Scale}

The severity of the main clinical symptoms (cough, rails, dyspnea) was evaluated using a severity scale we designed (Table E2, Online Repository), on which the assessment scores of the symptoms ranged from 0.5 (complete lack of symptoms) to 6 (maximal intensity). The severity of the disease was scored before the initiation of rituximab/abatacept therapy and again after 3, 6, and 12 months of treatment. 
The initial radiological ILLD symptoms and their dynamics during treatment were evaluated using multi-slice computed tomography (CT). Because there are no standard criteria for radiological evaluation of ILLD symptoms $(23,24)$, we developed an algorithm for use in this CT-based evaluation (Table E3, Supplementary Material). The following changes were scored: focal lesions, presence of patches of diffuse ground-glass opacity indicative of interstitial changes, linear and reticular changes of interlobar interstitium or fibrosis, lobe pneumatization, and hilar/mediastinal lymph node size. Each sign was assigned a point score ranging from 0 to 3 , each lung lobe was assessed separately, and the maximum possible number of points was 49 .

The CT changes characteristic of ILLD varied among patients and included three major types of lesions: ground-glass opacities or cotton-like foci, which are typical of FB; large sarcoid granuloma-like foci, typical of NLH; and diffuse interstitial changes, typical of LIP (Figure E1, Supplementary Material).

Due to the age restriction and the disease clinical manifestations in some patients (i.e., myopathy and polyneuropathy in AT patients), spirometry was not performed in the majority of patients; therefore, its results were not included in the analysis.

The parameters of the blood gases were evaluated using the standard method and an ABL800 Flex blood gas analyzer (Radiometer, Denmark).

\section{Morphology and Immunohistochemistry and Therapy Tactics}

The lung tissue pathomorphology was evaluated using standard $\mathrm{H} \& \mathrm{E}$ staining, and immunohistochemistry was evaluated using a set of markers that included CD20, Pax5, CD3, CD4, CD8, TdT, and Ki67. In all cases, in situ hybridization for the Epstein-Barr virus (EBV) marker EBER ISH Blue was performed.

To verify the ILLD diagnosis and define its form, a thoracoscopic lung biopsy was performed for most (22/29) patients. In the seven patients in whom biopsy was not possible due to the high risks associated with the procedure and/or the refusal of the parents, the ILLD form had been assigned based only on the radiological changes. The following pathomorphological forms of ILLD were identified: NLH (3/29), LIP (8/29), mixed changes characteristic of both FB and NLH (12/29), and FB and LIP (6/29) (Table E1, Supplementary Material).

According to the immunohistology, in all cases of abnormal BALT follicular hyperplasia (seen in both FB and NLH), predominantly B-cell $(\mathrm{CD} 20+)$ proliferation was noted, while the diffuse interstitial changes seen in LIP were associated predominantly with T-cell (CD3+) infiltration.

Based on the predominant type of lymphocytic infiltration of the lungs ( $\mathrm{B}$ or $\mathrm{T}$ lymphocytes), patients received either antiCD20 therapy directed at mature B-cells (rituximab) or the T-cell costimulatory molecule inhibitor abatacept. For the analysis, patients were divided into two groups according to the treatment received.

Group 1 patients received rituximab at $375 \mathrm{mg} / \mathrm{m}^{2}$ as 4 weekly, consecutive intravenous (IV) infusions, with subsequent infusions of $375 \mathrm{mg} / \mathrm{m}^{2}$ performed every 3 months for 12 months.

Group 2 patients received abatacept at $10 \mathrm{mg} / \mathrm{kg}$ as 2 biweekly IV infusions, and then every 4 weeks for 12 months.
Four patients (P2, P4, P7, and P11) originally treated using rituximab (Group 1) reached only partial remission. Later, they were treated using abatacept. These patients were analyzed in both Groups 1 and 2, and their ILLD symptoms were assessed at the beginning of each respective treatment.

\section{Quality of Life Evaluation}

The patients' quality of life was evaluated using an adapted Russian-language version of the Pediatric Quality of Life Inventory (PedsQL) 4.0 questionnaire both before the rituximab/abatacept treatment and after 12 months of therapy. Physical functioning (PF), psychosocial functioning (PSF), and total scale scores (TS) were evaluated.

\section{Statistical Analysis}

Statistical analysis of the data was performed using the XLSTAT Addinsoft 2017 software. The changes in the clinical and radiological signs were analyzed using the Mann-Whitney $U$ test. The difference was considered statistically significant if $\mathrm{p}<0.05$.

\section{Ethics Statement}

This retrospective study (ClinicalTrials.gov ID: NCT04572620) was approved by the Ethics Committee of the Dmitry Rogachev National Medical Research Center of Pediatric Hematology, Oncology, and Immunology.

\section{RESULTS}

Most patients were still in the process of genetic PID verification at the time of the ILLD diagnosis, therefore the choice of targeted preparation was primarily based on the histological and radiological findings. It was hypothesized that ILLD with predominant B lymphocyte infiltration will respond to rituximab. Yet, two patients with predominant $\mathrm{T}$ cell infiltration and LIP histology were previously treated with rituximab for other autoimmune complications and no ILLD improvement was noted. Hence it was hypothesized that abatacept might be a preparation of choice in the LIP group. Three patients with $\mathrm{NLH}, 12$ with $\mathrm{NLH}+\mathrm{FB}$ and one with $\mathrm{FB}+\mathrm{LIP}$ received treatment with rituximab, 8 patients with LIP and 10 with $\mathrm{FB}+\mathrm{LIP}$ received abatacept, and 4 patients with $\mathrm{NLH}+\mathrm{FB} / \mathrm{FB}+\mathrm{LIP}$ received consecutive treatment with rituximab and abatacept (Figure 1).

\section{Pathomorphological and CT Characteristics of ILLD}

Group 1 (Rituximab) consisted of 11 girls and 5 boys whose median age was 12 (6-18) years, and the PID/genetic defect distribution was as follows: del22 (3/16), NFkB1/2 (NFkB1/2) deficiency (2/16), CTLA4 (CTLA4) deficiency (1/16), Nijmegen syndrome $(N B S)(1 / 16)$, LRBA $(L R B A)$ deficiency $(1 / 16)$, Artemis deficiency (DCLRE1C) (1/16), and CID without genetic defect (7/16). 


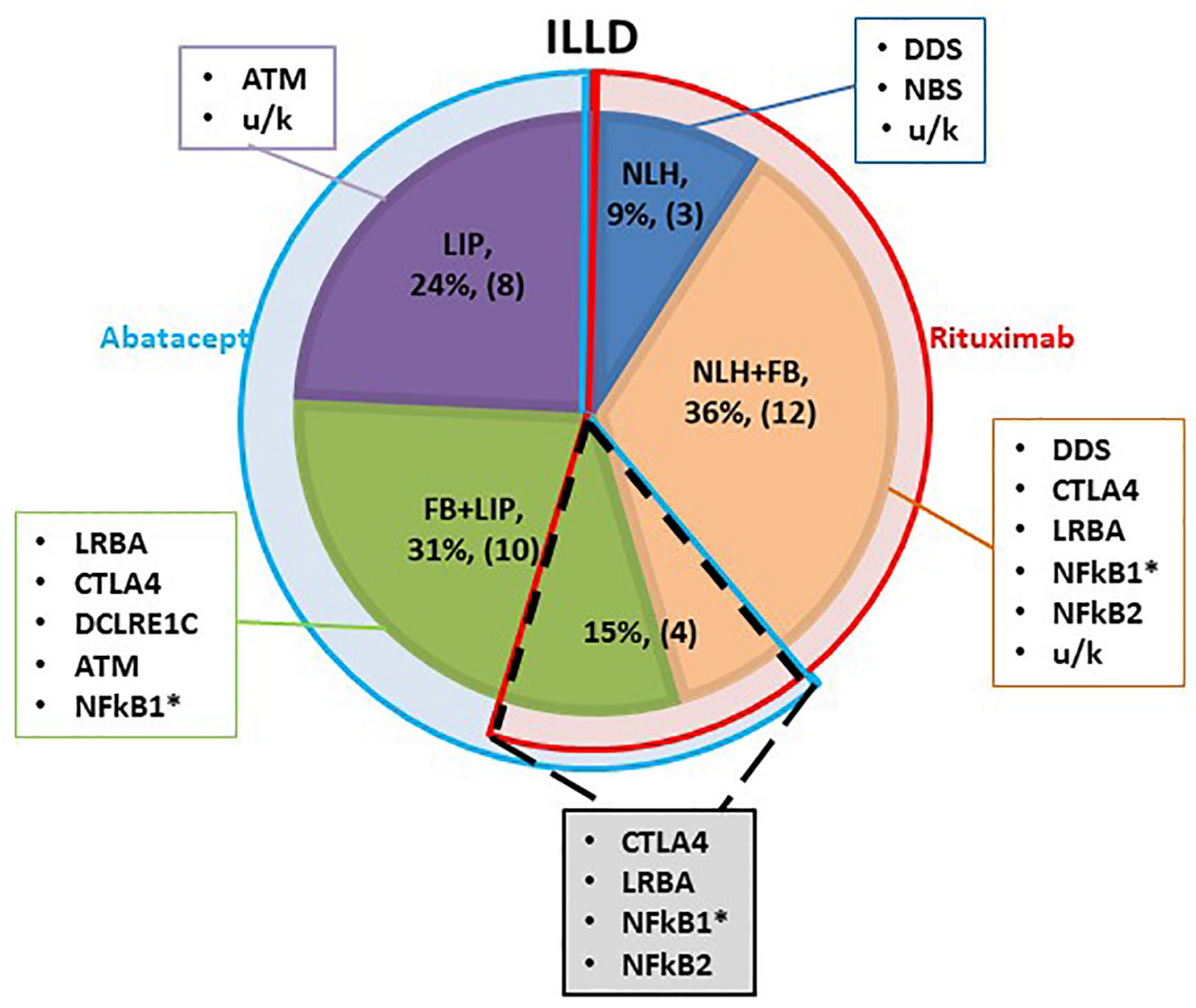

FIGURE 1 | Schematic characterization of the ILLD patient groups. Shaded segments represent different histological types of ILLD, with genetic PID defects noted in corresponding rectangles. Blue semi-ring denotes patients treated with abatacept, red - with rituximab. Overlapping segment (dotted line) denotes patients who were treated with rituximab, and later with abatacept. * - biopsy was performed twice.

The morphological and/or radiological changes in this group were characterized as a combination of FB and NLH $(12 / 16)$, NLH (3/16), and a combination of FB and LIP (1/16) (Table E1, Figure E1, Supplementary Material).

Group 2 (Abatacept) included 12 girls and 5 boys whose median age was 10 (5-17) years, and the PID/genetic defects distribution was as follows: AT (ATM) (8/17), CTLA4 deficiency (CTLA4) (4/ 17), LRBA deficiency ( $L R B A)$ (2/17), NFkB1/2 deficiency (NFkB1/ 2) $(2 / 17)$, and CID without genetic defect $(1 / 17)$.

The morphological and/or radiological changes in Group 2 were characterized as LIP (8/17) or a combination of LIP and FB (9/17) (Table E1, Figure E1, Supplementary Material).

\section{Dynamics of the Clinical and Radiological Symptoms of ILLD in Patients Treated With Rituximab (Group 1)}

Before initiation of rituximab, the symptoms of the patients in Group 1 varied in intensity from subclinical (3/16) to chronic respiratory failure (3/16), including Grade 1 in two patients and Grade 3 in one patient. At the baseline, the average disease severity was $2.8 \pm 1.0$, which improved significantly to $1.3 \pm$ 0.5 after 4 weeks of rituximab therapy $(p=0.03)$ and to $0.7 \pm$ 0.05 at 6 months $(\mathrm{p}<0.01)$ (Figure 2$)$. The average radiological score at baseline was $30 \pm 7.1$, which during treatment slowly improved to reach significance at 6 months, while severity decreased to $10 \pm 1.7$ by 12 months ( $\mathrm{p}<0.01)$ (Figures 2, 3).

Before therapy, abnormal blood gases and oxygen saturation $\left(\mathrm{SaO}_{2}\right)$ were observed in three patients who had respiratory failure. Before treatment, a minimal $\mathrm{SaO}_{2}$ value of $75 \%$ (average $\mathrm{SaO}_{2} 95 \pm 11 \%$; normal range 98-100\%) and $\mathrm{pCO}_{2}$ value of $55 \mathrm{~mm} \mathrm{Hg}$ (average $51 \pm 3.5 \mathrm{~mm} \mathrm{Hg}$; normal range 35$45 \mathrm{~mm} \mathrm{Hg}$ ) were observed in a patient who had Grade 3 respiratory failure, and the other two patients who had Grade 1 respiratory failure had $\mathrm{SaO} 2$ values of $95-96 \%$ and $\mathrm{pCO} 2$ values of $43-48 \mathrm{~mm} \mathrm{Hg}$. After 12 months of treatment, these values returned to normal ranges. 
After 12 months of treatment, full remission was reported in 9 patients, partial remission in 6 , and no effect in 1 patient. Of the six patients who achieved partial remission, two (P15, P16) had minimal CT findings of FB at 12 months and continued therapy with rituximab (Table 1). In the remaining four patients (P2, P4, P7, and P11) who originally had NLH+FB ILLD, all the NLH and most FB abnormalities resolved; however, radiological changes in the form of interstitial damage became obvious, and the patients were then classified as FB+LIP (Figure E2, Supplementary Material). These patients were switched to abatacept and achieved complete remissions (described in the next section). In one patient (P6) who had FB+LIP, rituximab had no effect on the ILLD symptoms. Due to the severity of the disease, this patient subsequently underwent HSCT and died from post-transplant complications.

\section{Dynamics of the Clinical and Radiological Symptoms in ILLD Patients Treated With Abatacept (Group 2)}

In contrast to Group 1, most patients in Group 2 (16/17) had evident respiratory symptoms, with an average clinical score of $3.4 \pm 1.3$. Grade 1 respiratory failure was present in three cases, and Grade 2 was present in one case. Only one patient had asymptomatic CT changes.

Patients in Group 2 demonstrated symptom changes that were much slower than in Group 1, especially regarding the radiological signs of ILLD. While their clinical symptoms, including cough, respiratory failure, and rails, improved after six months of abatacept therapy and the average score decreased from $1.2 \pm 0.3$ to $0.6 \pm 0.1$ ( $\mathrm{p}<0.01)$, their CT changes were even slower: Significant increases in lung pneumatization and the disappearance of or substantial decreases in the ground-glass symptoms, fibrosis, and peribronchial changes were observed only after 12 months of treatment, and the average score decreased from $18.4 \pm 3.1$ to $6.0 \pm 2.0(\mathrm{p}<0.01)$ (Figures 4, 5)

After 12 months of treatment, full remission was reported in all 17 patients (Table 1).

In four patients, abnormal $\mathrm{SaO}_{2}$ (average $93.5 \pm 0.9 \%$ ) and $\mathrm{pCO}_{2}$ values (average $52 \pm 4.7 \mathrm{~mm} \mathrm{Hg}$ ) and signs of respiratory failure were noted. These values normalized after six months of treatment.

Two patients from Group 2 (P17, P23) had previously received rituximab (four weekly doses) for other complications, including Coombs hemolytic anemia, lymphoproliferative syndrome, and persistent EBV-viremia. No improvements in their ILLD were noted after their short courses of treatment.

\section{Adverse Events}

No cases of therapy intolerance or serious adverse effects of rituximab or abatacept were recorded during treatment. During the first two rituximab infusions, four patients had fevers that
A

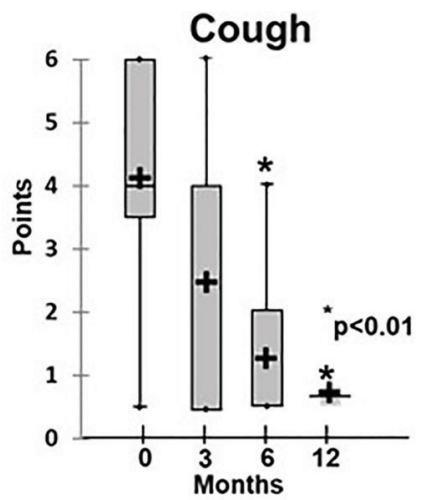

C

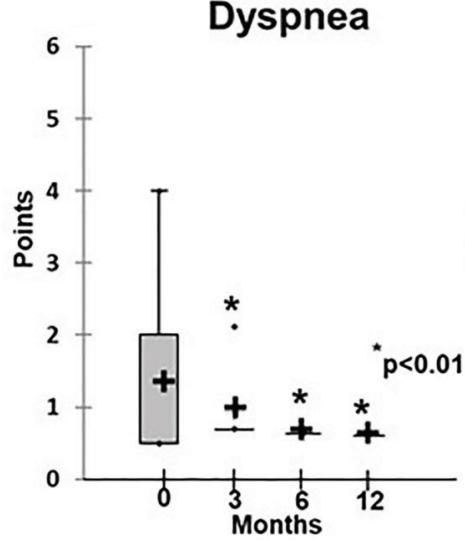

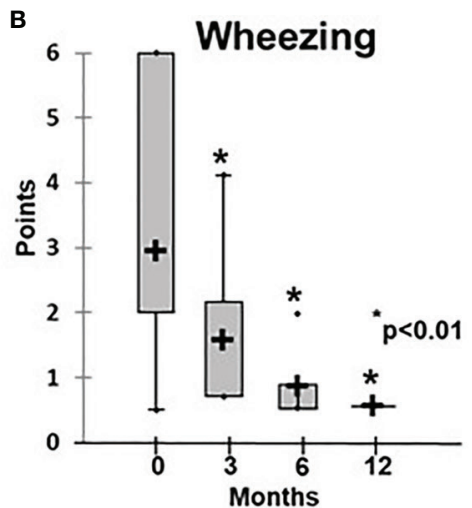

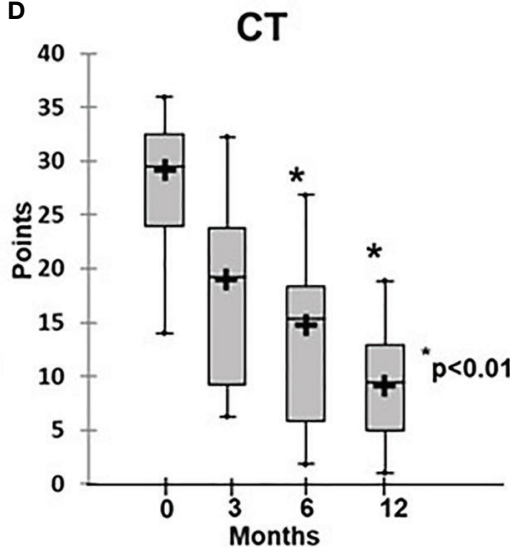

FIGURE 2 | Dynamics of clinical and radiological ILLD symptoms in patients treated with rituximab. (A) Cough, (B) wheezing, (C) dyspnea, (D) CT changes. 


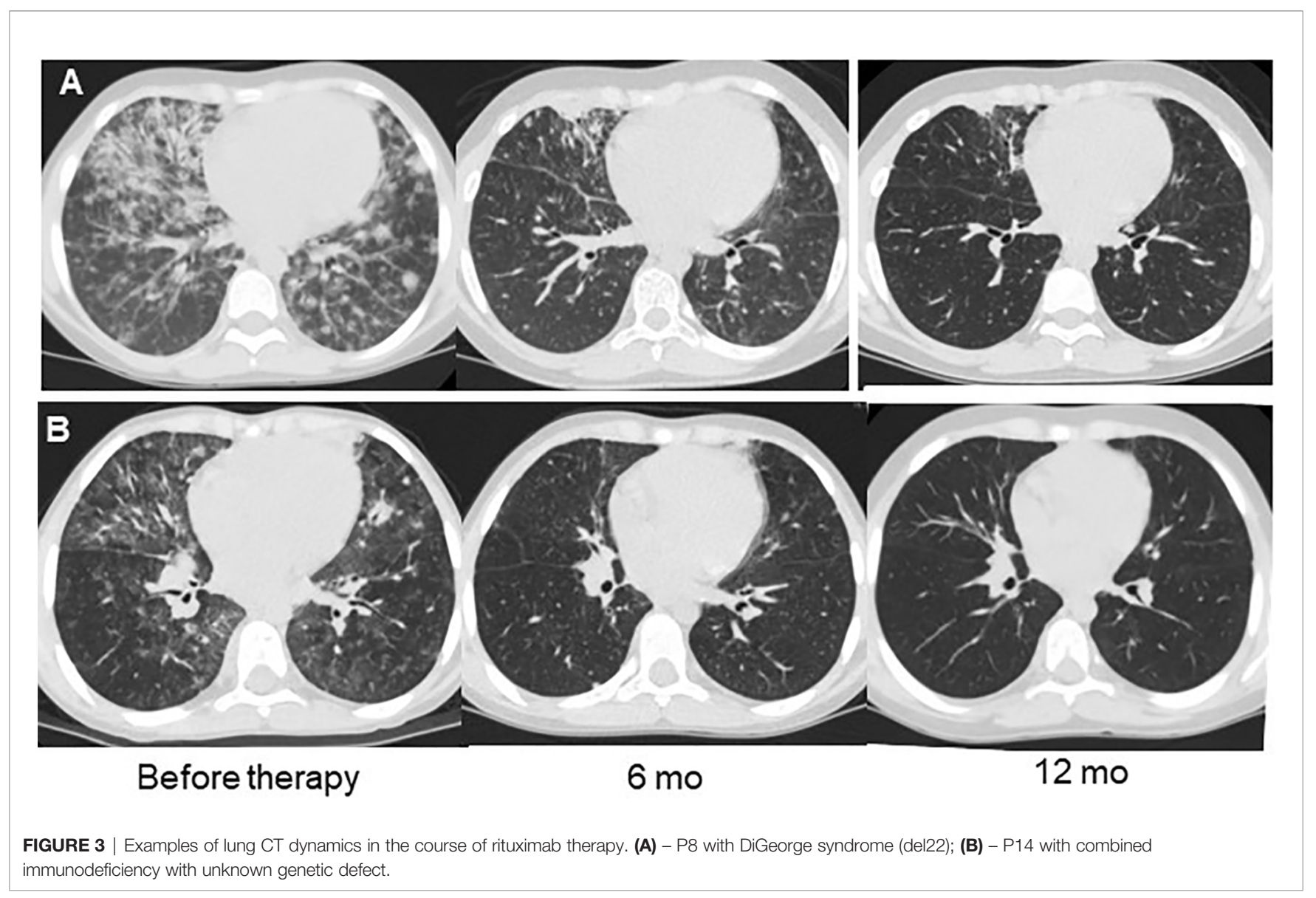

completely resolved after treatment with nonsteroid antiinflammatory drugs. These events constituted a rate of $6.7 \%$ of all rituximab infusions.

\section{Quality of Life}

The quality of life assessment demonstrated that patients who had PID and ILLD had physical and psychosocial function impairments: Before the treatment, the average TS score was $31.2 \pm 1.9$ points. The treatment provided statistically significant improvements to the physical and psychosocial aspects, leading to increases in specific and overall scores, with the TS score at 12 months being $57 \pm 2.1$ (Table 2 and Figure 6).

\section{Long-Term Follow-Up}

At the data censoring point, 21/29 patients were alive, 2 of them had undergone successful allogeneic HSCT and were free of symptoms, including ILLD symptoms, and 5 were lost to follow up after 12 or more months of therapy. Three patients died in the early posttransplant period due to non-ILLD-related complications after having received HSCT. Therefore, 8 patients in Group 1 and 11 patients in Group 2 were available for long-term follow-up analysis ( $>12$ months), with the median follow-up time being 43.5 months (14-56 months). From Group 1, 6/8 patients were off rituximab therapy and in complete ILLD remission, and $2 / 8$ were still receiving treatment (Table E1, Supplementary material). From

TABLE 1 | The ILLD variants and response to therapy in Groups 1 and 2.

\begin{tabular}{|c|c|c|c|c|c|}
\hline Variant of ILLD & Patients, n & Complete remission, $\mathbf{n}$ & Partial remission, $\mathbf{n}$ & No effect, $n$ & On preparation*, n \\
\hline \multicolumn{6}{|c|}{ Group I } \\
\hline NLH & 3 & 3 & 0 & 0 & 0 \\
\hline $\mathrm{FB}+\mathrm{NHL}$ & 12 & 6 & 6 & 0 & 2 \\
\hline FB+LIP & 1 & 0 & 0 & 1 & 0 \\
\hline \multicolumn{6}{|c|}{ Group ॥ } \\
\hline LIP & 8 & 8 & 0 & 0 & 4 \\
\hline FB+LIP & 9 & 9 & 0 & 0 & 6 \\
\hline
\end{tabular}

FB, follicular bronchiolitis; LIP, lymphoid interstitial pneumonia; NLH, nodular lymphoid hyperplasia. *Follow up $10 \pm 2$ mo, $n$, number of patients. 

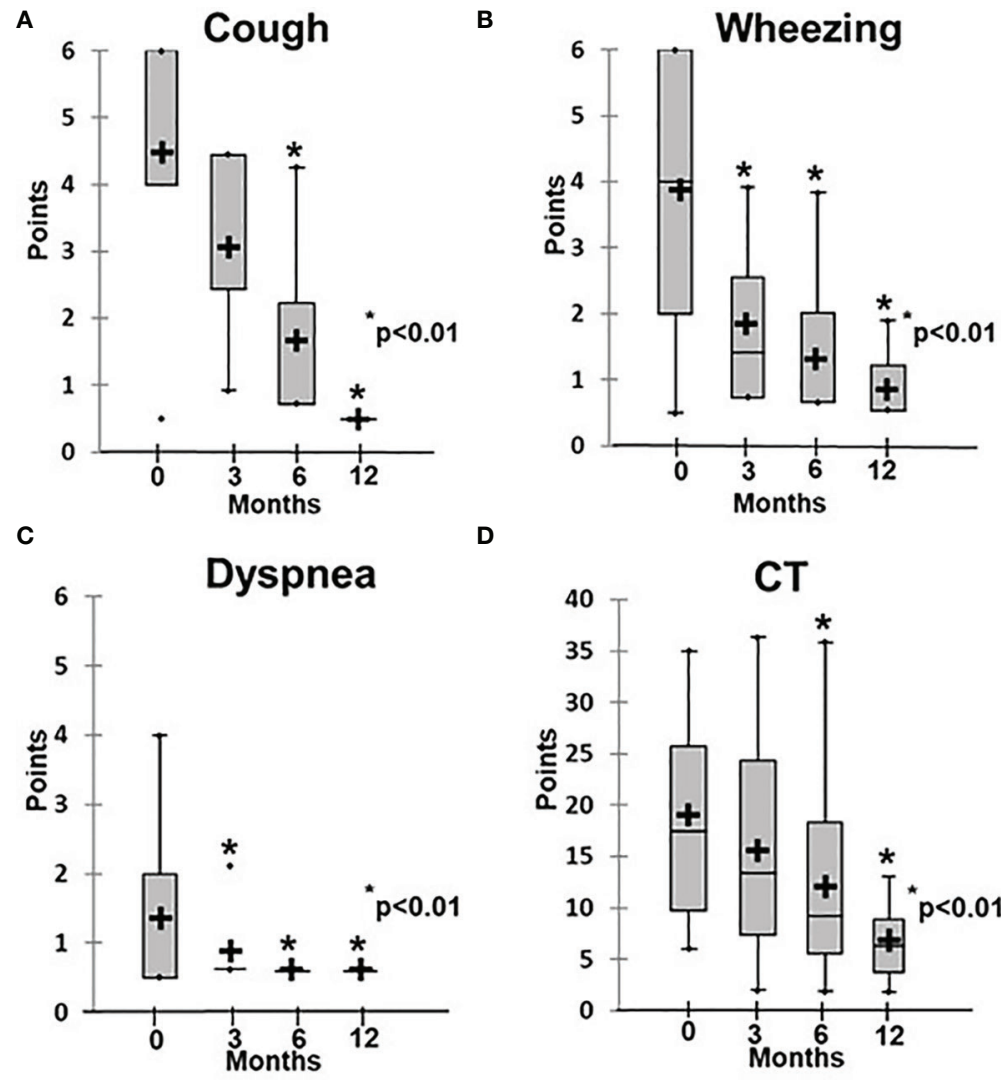

FIGURE 4 | Dynamics of clinical and radiological symptoms in patients treated with abatacept. (A) Cough, (B) wheezing, (C) dyspnea, (D) CT changes.

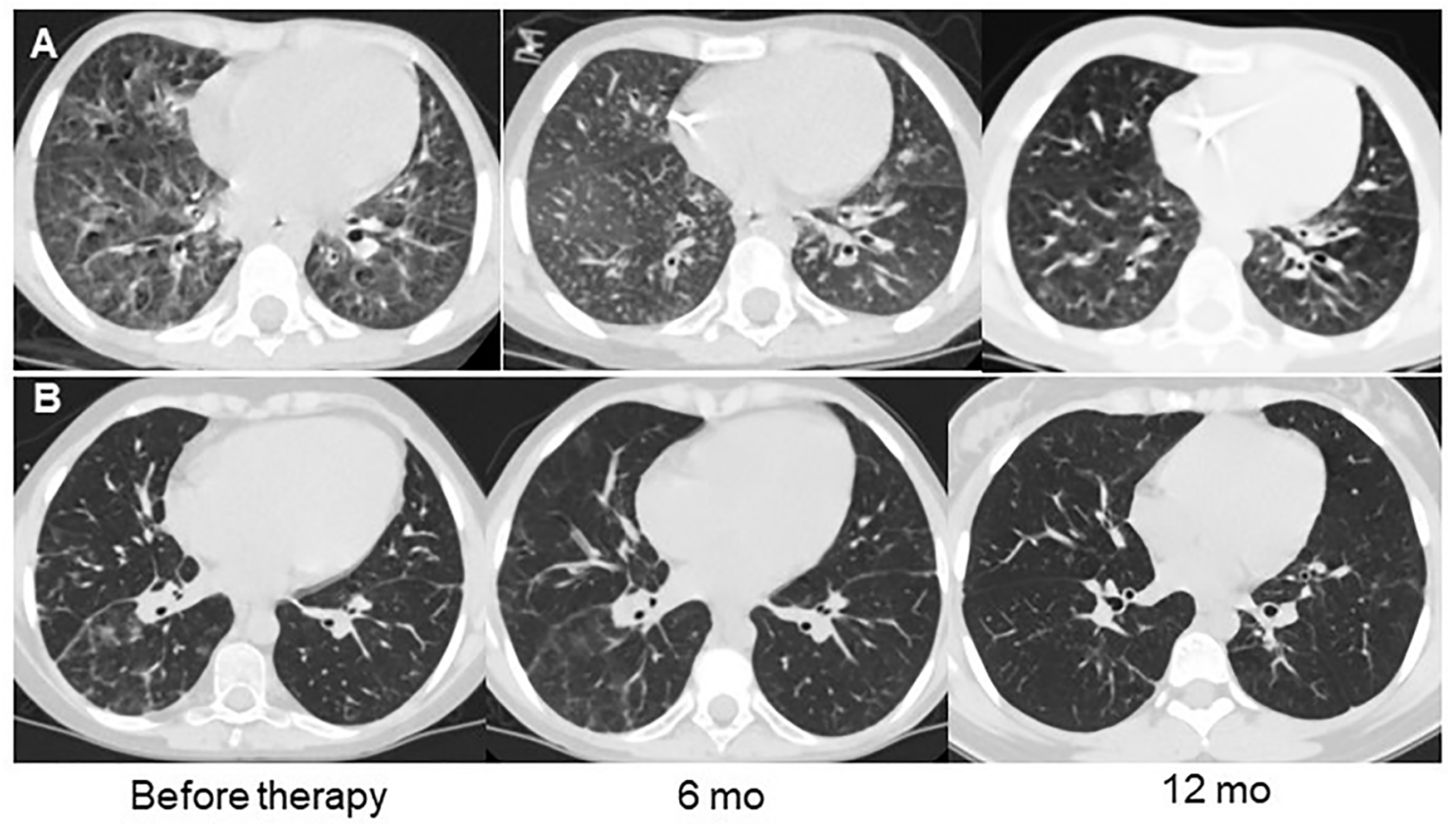

FIGURE 5 | Examples of lung CT dynamics in the course of abatacept therapy. (A) - P28 with CTLA4 deficiency, (B) - P27 with ataxia-telangiectasia. 
TABLE 2 | Results of the quality of life evaluation using PedsQL 4,0.

\begin{tabular}{|c|c|c|c|c|c|}
\hline & \multirow[t]{2}{*}{ PF score } & \multicolumn{3}{|c|}{ PSF score } & \multirow[t]{2}{*}{ TS score } \\
\hline & & EF & SF & CF & \\
\hline Prior to treatment & $31.1 \pm 2.7$ & $51.5 \pm 3.6$ & $30.4 \pm 3.6$ & $16.8 \pm 2.4$ & $31.2 \pm 1.9$ \\
\hline After 12 months of treatment & $51.2 \pm 3.7$ & $80.3 \pm 2.7$ & $52.4 \pm 3.8$ & $45.8 \pm 3.0$ & $57 \pm 2.1$ \\
\hline$P$ & $\mathrm{p}<0.001$ & $p<0.001$ & $p<0.001$ & $p<0.001$ & $p<0.001$ \\
\hline
\end{tabular}

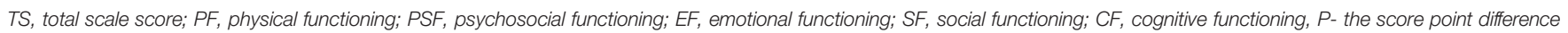
between initial value and the one obtained at the screening point is statistically significant $(p<0.01)$.

Group 2, 1/11 was off therapy with no signs of ILLD, and 10/11 were still receiving treatment. In three of these ten, attempts to discontinue abatacept treatment had resulted in the return of ILLD symptoms, and therapy had been re-established.

\section{DISCUSSION}

Recent years have been marked by the recognition of the interstitial lymphocytic lung disease as a severe complication of many PIDs. As reported in a consensus statement from specialists in internal diseases, pulmonology, and clinical immunology in the UK (25), ILLD is severely underdiagnosed in adult PID patients due to the lack of widely accepted protocols for its diagnosis and management. To our knowledge, this is the first published observation of ILLD in a large cohort of pediatric patients, so its prevalence in pediatric PID is even more difficult to assess. As in adult PID patients (14), the effect of "classic" immunosuppressive therapies (local and systemic steroids, azathioprine, methotrexate, sirolimus, mycophenolate mofetil, and adalimumab) in our ILLD cohort had been unsatisfactory. Therefore, new treatment modalities are required.

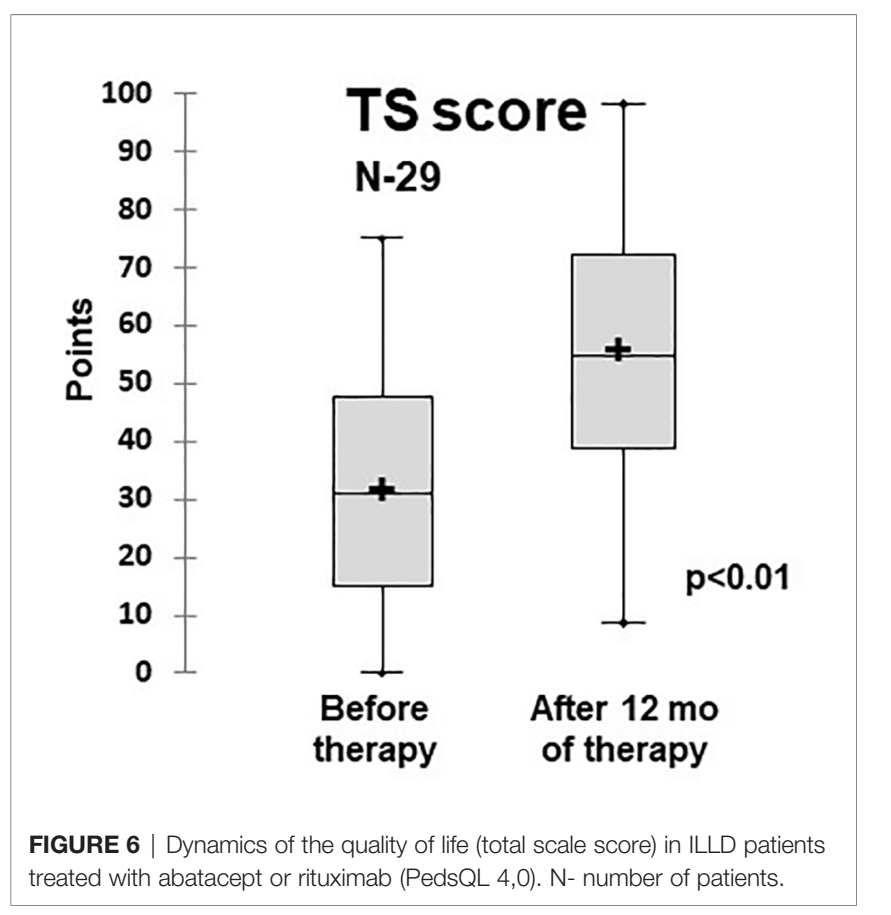

Immune dysregulation leading to impaired mucosal tolerance is considered the leading mechanism of ILLD development (11, 26). However, the radiological and histomorphological pictures of ILLD vary among patients, making it difficult to classify for diagnostic and treatment purposes (27). As suggested previously, the cellular composition of the hyperplastic BALT and interstitial infiltrates might provide the basis for such a classification, as well as for the stratification of targeted ILLD treatments (28).

The patients analyzed in the current study were differentially treated with abatacept or rituximab, based predominantly on the histologically proven or radiologically predicted prevalence of $\mathrm{T}$ or B-lymphocytes, respectively, in their lung infiltrates.

We demonstrated the dramatic effects of both treatments in their respective groups. In rituximab recipients, the response was observed after the very first month of treatment, while in abatacept recipients, significant clinical and radiological improvements were observed only after six months of therapy or even more. In our opinion, this may be due to the more extensive lung damage in LIP as compared to the focal lung lesions found in FB and NLH patients. In addition, the rituximab dose used in our patients was derived from the B-cell lymphoma treatment protocols aimed at the rapid elimination of tumor B-cells $(29,30)$, whereas the abatacept dose was initially developed to treat rheumatoid arthritis (31) and might not have been high enough to rapidly control the immune-mediated damage in patients with inherited immune dysregulation syndromes. We suggest that further studies aim to optimize the abatacept doses and regimens used to treat immune dysregulation in general and ILLD in particular.

We also suggest that in addition to histopathology, the response to ILLD therapy is determined by the underlying genetic defect. In our cohort, the most aggressive ILLD course was seen in patients who had profound T-cell dysregulation defects (CTLA, NFkb1/2, and $L R B A$ deficiencies) and in AT patients, which is in accordance with the results of previous studies $(7,21,22,32)$. Four patients who had immune dysregulation PID were originally treated and responded quickly, but only partially to rituximab. All were subsequently treated with abatacept and achieved full ILLD remission. More studies are needed to investigate if abatacept can be used as a monotherapy in cases of ILLD with histological findings of $\mathrm{NLH}$ or $\mathrm{NLH}+\mathrm{FB}$ and underlying defects of immune dysregulation (for instance, CTLA or LRBA deficiency), or a course of rituximab is initially required to quickly reduce the pathology caused by B cell hyperproliferation in these cases.

Due to the retrospective character the study had certain limitations. For instance, most patients did not receive treatment with both abatacept and rituximab to prove superiority of one 
treatment over the other based on the pathomorphological approach. Yet, two patients with LIP histopathology had previously received rituximab, and no improvement in the course of ILLD was noted, whereas abatacept treatment was subsequently successful. In our opinion it is highly suggestive that LIP changes do not respond to the B cell targeted treatment.

There is also a question regarding the optimal duration of treatment and/or long-term therapeutic modification. In our study, while rituximab was discontinued in most patients successfully, without any ILLD relapse, most patients in whom abatacept treatment was discontinued demonstrated ILLD progression and required continuous treatment. These were mostly patients who had AT, for which no curative treatment is currently available. Hence, the efficacy and safety of long-term-potentially lifelong-abatacept treatment require further investigation.

In the current study, treatment with either abatacept or rituximab was accompanied by only a few adverse effects, which were reversible. This suggests that these regimens are not only clinically effective but also safe, in stark contrast to the preceding steroid treatment, which had been accompanied by significant side effects.

Many patients in our group had predominantly radiological symptoms at the time of their ILLD diagnoses. Yet, given that other patients, some as young as 2.5 years old, already had signs of respiratory failure due to ILLD, awareness of immune lung diseases in children who have PID, early diagnosis, and early treatment are required to prevent severe lung damage and to improve the quality of life, which we determined to be significantly affected by ILLD.

In conclusion, the use of abatacept and rituximab as targeted monotherapies demonstrated sufficient efficacy and safety in the treatment of children who had ILLD-complicated PID. Questions of the optimal therapy duration and/or modification of the treatment regimen, which have been raised by this and previous studies, should be resolved based on the results of longer-term follow-up and subsequent prospective studies.

\section{DATA AVAILABILITY STATEMENT}

The original contributions presented in the study are included in the article/Supplementary Material. Further inquiries can be directed to the corresponding author.

\section{REFERENCES}

1. Bousfiha A, Jeddane L, Picard C, Al-Herz W, Ailal F, Casanova JL, et al. Human Inborn Errors of Immunity: 2019 Update of the IUIS Phenotypical Classification. J Clin Immunol (2020) 40(1):66-81. doi: 10.1007/s10875-020-00758-x

2. Lehman HK. Autoimmunity and Immune Dysregulation in Primary Immune Deficiency Disorders. Curr Allergy Asthma Rep (2015) 15(9):53-62. doi: 10.1007/s11882-015-0553-x

3. Walter JE, Ayala IA, Milojevic D. Autoimmunity as a Continuum in Primary Immunodeficiency. Curr Opin Pediatr (2019) 31(6):851-62. doi: 10.1097/ MOP.0000000000000833

4. Guinee DG Jr. Update on Nonneoplastic Pulmonary Lymphoproliferative Disorders and Related Entities. Arch Pathol Lab Med (2010) 134:691-701. doi: 10.1043/1543-2165-134.5.691

5. Morimoto Y, Routes JM. Granulomatous Disease in Common Variable Immunodeficiency. Curr Allergy Asthma Rep (2005) 5(5):370-5. doi: 10.1007/ s11882-005-0008-x

\section{ETHICS STATEMENT}

The studies involving human participants were reviewed and approved by The Ethics Committee of the Dmitry Rogachev National Medical Research Center of Pediatric Hematology, Oncology, and Immunology. Written informed consent to participate in this study was provided by the participants' legal guardian/next of kin.

\section{AUTHOR CONTRIBUTIONS}

All authors contributed to the article and approved the submitted version. AS drafted the manuscript, and contributed to concept and design, critical revision of the intellectual content, and final approval. GN contributed to concept and design, critical revision of the intellectual content, and final approval YR wrote the manuscript, created the tables and figures. DA performed pathomorphological studies. GT design of radiological ILLD severity evaluation scale and analyzed radiological data. ED, OS, AR, DY, AL, VB provided clinical care. AM performed statistical analyses.

\section{ACKNOWLEDGMENTS}

The authors would like to thank the anesthesiology and surgery teams of NRC PHOI for performing thoracoscopic lung biopsies, our colleagues from cytogenetics and molecular biology laboratories for the molecular genetic tests performed and, of course, our patients and their caregivers for their trust in us and resilience in the face of the disease.

\section{SUPPLEMENTARY MATERIAL}

The Supplementary Material for this article can be found online at: https://www.frontiersin.org/articles/10.3389/fimmu.2021.704261/ full\#supplementary-material

6. Schubert D, Bode C, Kenefeck R, Hou TZ, Wing JB, Kennedy A, et al. Autosomal Dominant Immune Dysregulation Syndrome in Humans With CTLA4 Mutations. Nat Med (2014) 20(12):1410-6. doi: 10.1038/nm.3746

7. Gamez-Diaz L, August D, Stepensky P, Revel-Vilk S, Seidel MG, Noriko M, et al. The Extended Phenotype of LPS-Responsive Beige-Like Anchor Protein (LRBA) Deficiency. J Allergy Clin Immunol (2016) 137:223-30. doi: 10.1016/j.jaci.2015.09.025

8. de Gracia J, Vendrell M, Alvarez A, Pallisa E, Rodrigo MJ, de la Rosa D, et al. Immunoglobulin Therapy to Control Lung Damage in Patients With Common Variable Immunodeficiency. Int Immunopharmacol (2004) 4 (6):745-53. doi: 10.1016/j.intimp.2004.02.011

9. Lucas M, Lee M, Lortan J, Lopez-Granados E, Misbah S, Chapel H. Infection Outcomes in Patients With Common Variable Immunodeficiency Disorders: Relationship to Immunoglobulin Therapy Over 22 Years. J Allergy Clin Immunol (2010) 125:1354-60. doi: 10.1016/j.jaci.2010.02.040

10. Poletti V, Ravaglia C, Tomassetti S, Gurioli C, Casoni G, Asioli S, et al. Lymphoproliferative Lung Disorders: Clinicopathological Aspects. Eur Respir $\operatorname{Rev}(2013)$ 22:427-36. doi: 10.1183/09059180.00004313 
11. Maglione PJ, Ko HM, Beasley MB, Strauchen JA, Cunningham-Rundles C. Tertiary Lymphoid Neogenesis is a Component of Pulmonary Lymphoid Hyperplasia in Patients With Common Variable Immunodeficiency. J Allergy Clin Immunol (2014) 133:535-42. doi: 10.1016/j.jaci.2013.08.022

12. Bates CA, Ellison MC, Lynch DA, Cool CD, Brown KK, Routes JM. Granulomatous-Lymphocytic Lung Disease Shortens Survival in Common Variable Immunodeficiency. J Allergy Clin Immunol (2004) 114:415-21. doi: 10.1016/j.jaci.2004.05.057

13. Chapel H, Cunningham-Rundles C. Update in Understanding Common Variable Immunodeficiency Disorders (CVIDs) and the Management of Patients With These Conditions. Br J Haematol (2009) 145(6):709-27. doi: 10.1111/j.1365-2141.2009.07669.x

14. Boursiquot J-N, Gérard L, Malphettes M, Fieschi C, Galicier L, Boutboul D, et al. Granulomatous Disease in CVID: Retrospective Analysis of Clinical Characteristics and Treatment Efficacy in a Cohort of 59 Patients. J Clin Immunol (2013) 33:84-95. doi: 10.1007/s10875-012-9778-9

15. Davies CW, Juniper MC, Gray W, Gleeson FV, Chapel HM, Davies RJ. Lymphoid Interstitial Pneumonitis Associated With Common Variable Hypogammaglobulinaemia Treated With Cyclosporin a. Thorax (2000) 55:88-90. doi: 10.1136/thorax.55.1.88

16. Thatayatikom A, Thatayatikom S, White AJ. Infliximab Treatment for Severe Granulomatous Disease in Common Variable Immunodeficiency: A Case Report and Review of the Literature. Ann Allergy Asthma Immunol (2005) 95:293-300. doi: 10.1016/S1081-1206(10)61228-8

17. Franxman TJ, Howe LE, Baker JR Jr. Infliximab for Treatment of Granulomatous Disease in Patients With Common Variable Immunodeficiency. J Clin Immunol (2014) 34:820-7. doi: 10.1007/s10875-014-0079-3

18. Chase NM, Verbsky JW, Hintermeyer MK, Waukau JK, Tomita-Mitchell A, Casper JT, et al. Use of Combination Chemotherapy for Treatment of Granulomatous and Lymphocytic Interstitial Lung Disease (GLILD) in Patients With Common Variable Immunodeficiency (CVID). J Clin Immunol (2013) 33:30-9. doi: 10.1007/s10875-012-9755-3

19. Pathria M, Urbine D, Zumberg MS, Guarderas J. Management of Granulomatous Lymphocytic Interstitial Lung Disease in a Patient With Common Variable Immune Deficiency. BMJ Case Rep (2016) 2016: bcr2016215624. doi: 10.1136/bcr-2016-215624

20. Schwab C, Gabrysch A, Olbrich P, Patiño V, Warnatz K, Wolff D, et al. Phenotype, Penetrance, and Treatment of 133 Cytotoxic T-Lymphocyte Antigen 4-Insufficient Subjects. J Allergy Clin Immunol (2018) 142(6):193246. doi: 10.1016/j.jaci.2018.02.055

21. Kiykim A, Ogulur I, Dursun E, Charbonnier LM, Nain E, Cekic S, et al. Abatacept as a Long-Term Targeted Therapy for LRBA Deficiency. J Allergy Clin Immunol Pract Nov-Dec (2019) 7(8):2790-800. doi: 10.1016/ j.jaip.2019.06.011

22. ESID Registry. Working Definitions for Clinical Diagnosis of PID (Accessed February 28, 2021).

23. Jesenak M, Banovcin P, Jesenakova B, Babusikova E. Pulmonary Manifestations of Primary Immunodeficiency Disorders in Children. Front Pediatr (2014) 72:77. doi: 10.3389/fped.2014.00077

24. Torigian DA, LaRosa DF, Levinson AI, Litzky LA, Miller WT Jr. Granulomatous-Lymphocytic Interstitial Lung Disease Associated With Common Variable Immunodeficiency: CT Findings. J Thorac Imaging (2008) 23:162-9. doi: 10.1097/RTI.0b013e318166d32f
25. Hurst JR, Verma N, Lowe D, Baxendale HE, Jolles S, Kelleher P, et al. British Lung Foundation/United Kingdom Primary Immunodeficiency Network Consensus Statement on the Definition, Diagnosis, and Management of Granulomatous-Lymphocytic Interstitial Lung Disease in Common Variable Immunodeficiency Disorders. J Allergy Clin Immunol Pract (2017) 5:938-45. doi: 10.1016/j.jaip.2017.01.021

26. Arandi N, Mirshafiey A, Jeddi-Tehrani M, Abolhassani H, Sadeghi B, Mirminachi B, et al. Evaluation of CD4+CD25+FOXP3+ Regulatory T Cells Function in Patients With Common Variable Immunodeficiency. Cell Immunol (2013) 28:129-33. doi: 10.1016/j.cellimm.2013.03.003

27. Patel S, Anzilotti C, Lucas M, Moore N, Chapel H. Interstitial Lung Disease in Patients With Common Variable Immunodeficiency Disorders: Several Different Pathologies? Clin Exp Immunol (2019) 198(2):212-23. doi: $10.1111 /$ cei. 13343

28. Rao N, Mackinnon CA, Routes JM. Granulomatous and Lymphocytic Interstitial Lung Disease (GLILD): A Spectrum of Pulmonary Histopathological Lesions in Common Variable Immunodeficiency (CVID) - Histological and Immunohistochemical Analysis of 16 Cases. Hum Pathol (2015) 46(9):1306-14. doi: 10.1016/j.humpath.2015.05.011

29. Pierpont TM, Limper CB, Richards KL. Past, Present, and Future of Rituximab-The World's First Oncology Monoclonal Antibody Therapy. Front Oncol (2018) 8:163. doi: 10.3389/fonc.2018.00163

30. NICE. NG52 Non-Hodgkin's Lymphoma:Diagnosis and Management (2016). Available at: http://www.nice.org.uk/guidance/ng52 (Accessed February 28, 2021).

31. Chitale S, Moots R. Abatacept: The First T Lymphocyte Co-Stimulation Modulator, for the Treatment of Rheumatoid Arthritis. Expert Opin Biol Ther (2008) 8:115-22. doi: 10.1517/14712598.8.1.115

32. Bhatt JM, Bush A, van Gerven M, Nissenkorn A, Renke M, Yarlett L, et al. ERS Statement on the Multidisciplinary Respiratory Management of Ataxia Telangiectasia. Eur Respir Rev (2015) 24:565-81. doi: 10.1183/16000617. 0066-2015

Conflict of Interest: The authors declare that the research was conducted in the absence of any commercial or financial relationships that could be construed as a potential conflict of interest.

The handling editor declared a past collaboration with one of the authors, AR.

Publisher's Note: All claims expressed in this article are solely those of the authors and do not necessarily represent those of their affiliated organizations, or those of the publisher, the editors and the reviewers. Any product that may be evaluated in this article, or claim that may be made by its manufacturer, is not guaranteed or endorsed by the publisher.

Copyright $\odot 2021$ Rodina, Deripapa, Shvets, Mukhina, Roppelt, Yuhacheva, Laberko, Burlakov, Abramov, Tereshchenko, Novichkova and Shcherbina. This is an openaccess article distributed under the terms of the Creative Commons Attribution License (CC BY). The use, distribution or reproduction in other forums is permitted, provided the original author(s) and the copyright owner(s) are credited and that the original publication in this journal is cited, in accordance with accepted academic practice. No use, distribution or reproduction is permitted which does not comply with these terms. 